\title{
A simple two-gene prognostic model for adenocarcinoma of the lung
}

\author{
Carolyn E. Reed, MD, ${ }^{\text {a } A m a n d a ~ G r a h a m, ~ M S, ~}{ }^{a}$ Rana S. Hoda, MD, ${ }^{b}$ Andras Khoor, MD, ${ }^{d}$ Elizabeth Garrett-Mayer, PhD, ${ }^{\mathrm{c}}$ \\ Michael B. Wallace, $\mathrm{MD}^{\mathrm{e}}$ and Michael Mitas, $\mathrm{PhD}^{\mathrm{a}}$
}

From the Departments of Surgery, ${ }^{\text {a }}$ Pathology and Laboratory Medicine, ${ }^{\mathrm{b}}$ and Biostatics, Bioinformatics \& Epidemiology, Medical University of South Carolina, Charleston, SC, and the Department of Laboratory Medicine \& Pathology ${ }^{\mathrm{d}}$ and the Division of Gastroenterology \& Hepatology, Mayo Clinic, Jacksonville, Fla.

Read at the Eighty-seventh Annual Meeting of The American Association for Thoracic Surgery, Washington, DC, May 5-9, 2007.

Received for publication April 23, 2007; revisions received Sept 3, 2007; accepted for publication Oct 26, 2007.

Address for reprints: Carolyn E. Reed, MD, Medical University of South Carolina, 96 Jonathan Lucas St, 418 CSB, Charleston, SC 29425 (E-mail: reedce@musc.edu).

J Thorac Cardiovasc Surg 2008; 135:627-34 $0022-5223 / \$ 34.00$

Copyright $(2008$ by The American Association for Thoracic Surgery

doi:10.1016/j.jtcvs.2007.10.058
Objective: We hypothesized that clinical outcome of resected early-stage adenocarcinoma of the lung can be predicted by the expression of a few critically important genes as measured by quantitative real-time reverse-transcriptase polymerase chain reaction in formalin-fixed paraffin-embedded primary tumors.

Methods: Twenty-two prognostic genes for the metastatic phenotype were identified through complementary DNA microarray analysis of 4 cancer cell lines and bioinformatics analysis. Expression levels of a subset of these genes $(n=13)$ were measured by real-time time reverse-transcriptase polymerase chain reaction in formalin-fixed paraffin-embedded primary adenocarcinoma from patients whose disease recurred within 2 years $(n=9)$ and in patients who did not have a recurrence $(n=11)$. $R e-$ ceiver operating characteristic curves were analyzed to establish prognostic values of single genes. The most informative gene was combined with the remaining genes to determine whether there was a particular pair(s) that yielded high diagnostic accuracy. A small validation study was performed.

Results: Receiver operating characteristic curve analysis of the single genes revealed that high expression of CK19 was associated with nonrecurrence (area under the curve $=0.859$, confidence interval $=0.651-0.970$ ). The CK19/EpCAM2 gene ratio had the most reproducible prognostic accuracy, followed by the CK19/P-cadherin ratio. A Kaplan-Meier survival analysis generated from the CK19/EpCAM2 ratio resulted in highly significant curves as a function of marker positivity $(P=.0007$; hazard ratio $=10.7$ ). Significance declined but was maintained in the validation study.

Conclusions: This preliminary study provides evidence that the CK19/EpCAM2 and/ or CK19/P-cadherin ratio(s) may be a simple and accurate prognostic indicator of clinical outcome in early-stage adenocarcinoma of the lung. If further validation studies from large patient cohorts confirm the results, adjuvant therapy could be targeted to this high-risk group.

$\mathrm{D}$ espite surgical resection, patients with pathologic stage I non-small cell lung cancer (NSCLC) will have an approximately $30 \%$ to $40 \%$ incidence of recurrence and those with stage II a $45 \%$ to $60 \%$ recurrence rate. ${ }^{1}$ At present, the standard of care is to administer postoperative adjuvant chemotherapy to those patients with stage II NSCLC. ${ }^{2,3}$ Although there was initial enthusiasm for administering adjuvant therapy to patients with resected stage IB NSCLC, recent data do not support this practice. ${ }^{3-5}$ However, subsets of patients with stage I disease could potentially benefit from further treatment to prevent recurrence; likewise, a method to predict which patients with stage II disease could avoid the unnecessary toxicity of chemotherapy would be helpful.

The development of metastatic disease is the most common cause of death among patients with NSCLC and results from dissemination of malignant cells. It is now recognized that the ability of cells to gain metastatic potential is an intrinsic property of the primary tumor, which is substantiated by the high correlations between clinical outcome and gene expression profiles of a variety of primary tumors. ${ }^{6,7}$ The ability to predict clinical outcome on the basis of analysis of primary tumors would allow 


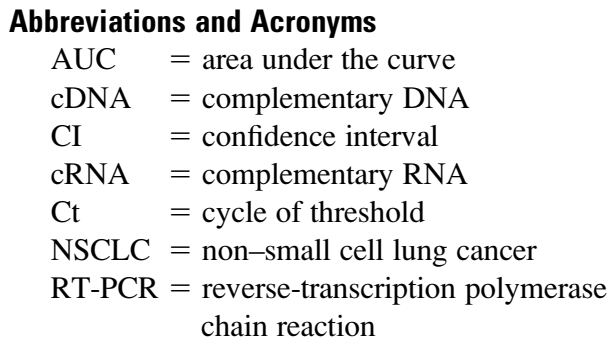

patients with cancer to be treated more effectively. However, the problem with many of these expression studies is that they require measurements of large sets of predictive genes using a platform (complementary DNA [cDNA] microarray analysis) that is not well suited to clinical application.

In this pilot study, we hypothesized that clinical outcome of patients with resected early-stage adenocarcinoma of the lung could be predicted by the expression of relatively few, but critically important, genes measured by quantitative real-time reverse-transcription polymerase chain reaction (RT-PCR) in formalin-fixed paraffin-embedded primary tumors. Specifically, we hypothesized that there exists a "good gene" and a "bad gene" such that the ratio of the two is a strong prognostic indicator of clinical outcome.

\section{Materials and Methods \\ Identification of 15 Highly Expressed Genes in NSCLC Cell Lines}

Expression levels of 22,283 gene transcripts were determined on oligonucleotide microarrays using RNA prepared from 4 NSCLC cell lines (CRL 5807 [bronchoalveolar carcinoma], CRL 5876 [adenocarcinoma derived from metastatic lymph node], A549 [adenocarcinoma], and HTB 177 (large cell carcinoma]), as well as from a pool of 4 normal cervical lymph nodes. Eight micrograms of total RNA per sample were used. First- and second-strand cDNA synthesis, double-stranded cDNA cleanup, biotin-labeled complementary RNA (cRNA) synthesis, cleanup, and fragmentation were performed according to protocols in the Affymetrix GeneChip Expression Analysis technical manual (Affymetrix, Santa Clara, Calif). Microarray analysis was performed by the DNA Microarray and Bioinformatics Core Facility at the Medical University of South Carolina using U133 A GeneChips (Affymetrix). Fluorescent images of hybridized microarrays were obtained by an HP GeneArray scanner (Affymetrix). For normalization, the microarray office suite was used such that all fluorescence values were multiplied by a factor that resulted in a mean fluorescent score for all genes equal to 150 . Data for normal lymph nodes were obtained from a previous study. ${ }^{8}$ All microarray results were imported into a single Microsoft Excel file. The first algorithm in the selection of highly expressed genes involved elimination of genes from NSCLC cell lines that were expressed in normal lymph nodes $(\mathrm{n}=11,326 ; 50.8 \%$ of total $[22,283])$. Of the remaining 10,957 genes, those that were detected in at least 2 NSCLC cell lines were first selected $(n=1731 ; 7.7 \%$ of total). After this round, genes whose mean fluorescence in all cell lines was greater than 500 were selected $(n=91 ; 0.41 \%$ of total). The final group of 91 genes was sorted according to mean cell line fluorescence/mean fluorescence of normal lymph nodes, and the 15 top genes were selected (Table 1).

\section{Bioinformatics Analysis to Identify Potentially Prognostic Genes in NSCLC}

Of the 15 most highly expressed genes identified by cDNA microanalysis, it was hypothesized that some were also expressed in other cancers, whereas some genes were specific for NSCLC. To identify

TABLE 1. Top 15 most highly overexpressed genes in lung cancer cell lines

\begin{tabular}{|c|c|c|c|c|c|c|c|}
\hline \multicolumn{3}{|c|}{ Gene description } & \multicolumn{5}{|c|}{ Affymetrix results* } \\
\hline & & & 1 & 2 & 3 & 4 & \\
\hline Rank & Gene & Acc. No. & A549 & HTB177 & CRL5807 & CRL5876 & Ratio $\dagger$ \\
\hline 1 & AGR2 & NM_006408 & 2124 & 2053 & 3082 & 38 & 960 \\
\hline 2 & S100P & NM_005980 & 242 & 2522 & 2673 & 4819 & 754 \\
\hline 3 & CK19 & NM_002276 & 27 & 935 & 1995 & 810 & 589 \\
\hline 4 & N001 & NM_000903 & 1375 & 1858 & 982 & 315 & 404 \\
\hline 5 & MET & NM_000245 & 1420 & 790 & 2429 & 378 & 348 \\
\hline 6 & MAGE-A6 & NM_005363 & 73 & 37 & 3004 & 4475 & 311 \\
\hline 7 & XAGE-1 & NM_020411 & 471 & 2 & 2322 & 3 & 250 \\
\hline 8 & KRTHB1 & NM_002281 & 2822 & 31 & 221 & 3 & 208 \\
\hline 9 & MAGE-A3 & NM_005362 & 116 & 29 & 4055 & 5107 & 178 \\
\hline 10 & MAP7 & NM_003980 & 455 & 466 & 381 & 930 & 116 \\
\hline 11 & AKR1B10 & NM_020299 & 11662 & 10603 & 17 & 75 & 101 \\
\hline 12 & CK7 related & NM_005556 & 537 & 21 & 1319 & 463 & 96 \\
\hline 13 & EpCAM2 & NM_002353 & 2 & 3 & 8146 & 2342 & 94 \\
\hline 14 & EpCAM1 & NM_002354 & 278 & 15 & 4430 & 3244 & 91 \\
\hline 15 & P-cadherin & NM_001793 & 2 & 3 & 1319 & 1274 & 87 \\
\hline
\end{tabular}

*Normalized fluorescent values obtained from Affymetrix U133A array data for the indicated cell line. †Ratio of mean NSCLC cell line data to mean of normal lymph node. 
genes that were highly expressed in other cancers, we queried the online Comparative Genome Anatomy Project (CGAP) National Cancer Institute 60-gene expression database (URL = http://cgap. nci.nih.gov) using all 15 genes. The output of a given query consists of a list of 10 genes whose expression levels are most highly correlated with the query sequence. Using the output of each gene, we constructed a correlation map such that the appearance of a gene on the map required (1) direct contact with one of the 15 highly expressed genes, (2) contacts with at least 2 genes, (3) that the correlation coefficient of any 2 genes must have a $P$ value $<8 \times 10^{-6}$, (4) that the relevant gene must be overexpressed in the CGAP SAGE data set in at least two cancers (with respect to normal tissue), and (5) that expression of the relevant gene must be at least 16 to 31 tags/ 200,000 sequenced tags in at least one cancer tissue. Genes identified from the first set of queries were used as query in a reiterative round of interrogation (data mining).

The correlation map obtained by this bioinformatics data mining approach contained a total of 22 genes (Figure 1). Seven of the 22 genes (AGR2, Map7, S100P, CK19, EpCAM1, EpCAM2, and Pcadherin) were derived from the list of 15 most highly expressed genes and are referred to as the primary prognostic genes (underlined in Figure 1). The remaining 15 genes identified from this bioinformatics approach are referred to as the secondary prognostic genes (italicized in Figure 1).

\section{Identification of Genes of Prognostic Value in Patients With Early-stage NSCLC Adenocarcinoma}

To determine whether the genes described above had potential prognostic value, we measured the expression levels by real-time RT-PCR in paraffin-embedded formalin-fixed primary tumors of patients whose adenocarcinoma recurred within 2 years (poor outcome group $\mathrm{A} ; \mathrm{n}=9$ ) and of patients who survived disease-free longer than 4 years (good outcome group $B ; n=11)$. Group A patients included 2 with stage IA, 2 stage IB, and 5 stage IIB disease. Group B patients included 5 with stage IA, 3 stage IB, and 3 stage IIB disease. Genes analyzed included the 7 primary prognostic genes, 6 second- ary prognostic genes (Sprint 2, Esx, CEA6, Ma12, GPX2, E-cadherin), as well as $\mu \mathrm{PAR}$, a gene whose expression has previously been shown to be associated with multiple cancers. The laboratory investigators were initially blinded to the clinical outcome. The study was approved by the Medical University of South Carolina Institutional Review Board.

A small validation study was performed using paraffin sections from patients with early-stage adenocarcinoma who had an early recurrence $(n=10)$ and survived greater than 2 years $(n=12)$ undergoing resection at the Mayo Clinic, Jacksonville, Florida.

Real-time RT-PCR of formalin-fixed paraffin-embedded samples was performed according to the method of Sprecht and associates. ${ }^{9}$ A $50-\mu \mathrm{m}$ section was cut from tissue blocks of primary tumor for messenger RNA extraction. For isolation of RNA, paraffinembedded tissue sections were deparaffinized twice with $1 \mathrm{~mL}$ of xylene at $37^{\circ} \mathrm{C}$ or room temperature for 10 minutes. The pellet was subsequently washed with $1 \mathrm{~mL}$ of $100 \%, 90 \%$, and $70 \%$ ethanol and air-dried at room temperature for 2 hours. The pellet was resuspended in $200 \mu \mathrm{L}$ of RNA lysis buffer (2\% lauryl sulfate, 10 $\mathrm{mmol} / \mathrm{L}$ Tris- $\mathrm{HCl}$ [pH 8.0], and $0.1 \mathrm{mmol} / \mathrm{L}$ ethylenediaminetetraacetic acid) and $100 \mu \mathrm{g}$ of proteinase $\mathrm{K}$ and incubated at $60^{\circ} \mathrm{C}$ for 16 hours. RNA was extracted by $1 \mathrm{~mL}$ of phenol/chloroform $(5: 1)$ solution (Sigma Chemical Company, St Louis, Mo). The aqueous layer containing RNA was transferred to a new 1.5-mL tube. Phenol/chloroform extraction was done a total of 3 times. RNA was precipitated with an equal volume of isopropanol, 0.1 volume of $3 \mathrm{~mol} /$ $\mathrm{L}$ sodium acetate, and $100 \mu \mathrm{g}$ of glycogen at $-20^{\circ} \mathrm{C}$ for 16 hours. After centrifugation at $12,000 \mathrm{rpm}$ for 15 minutes $\left(4^{\circ} \mathrm{C}\right)$, the RNA pellet was washed with $70 \%$ ethanol and air-dried at room temperature for 2 hours. Finally, the pellet was dissolved in $12 \mu \mathrm{L}$ of diethyl pyrocarbonate water. cDNA synthesis was performed with a panel of truncated gene-specific primers. Real-time RT-PCR was performed on a PE Biosystems Gene Amp 7300 or 7500 Sequence Detection System (PE Biosystems, Foster City, Calif). With the exception of the SYBR Green I master mix (purchased from Qiagen, Valencia, Calif), all reaction components were

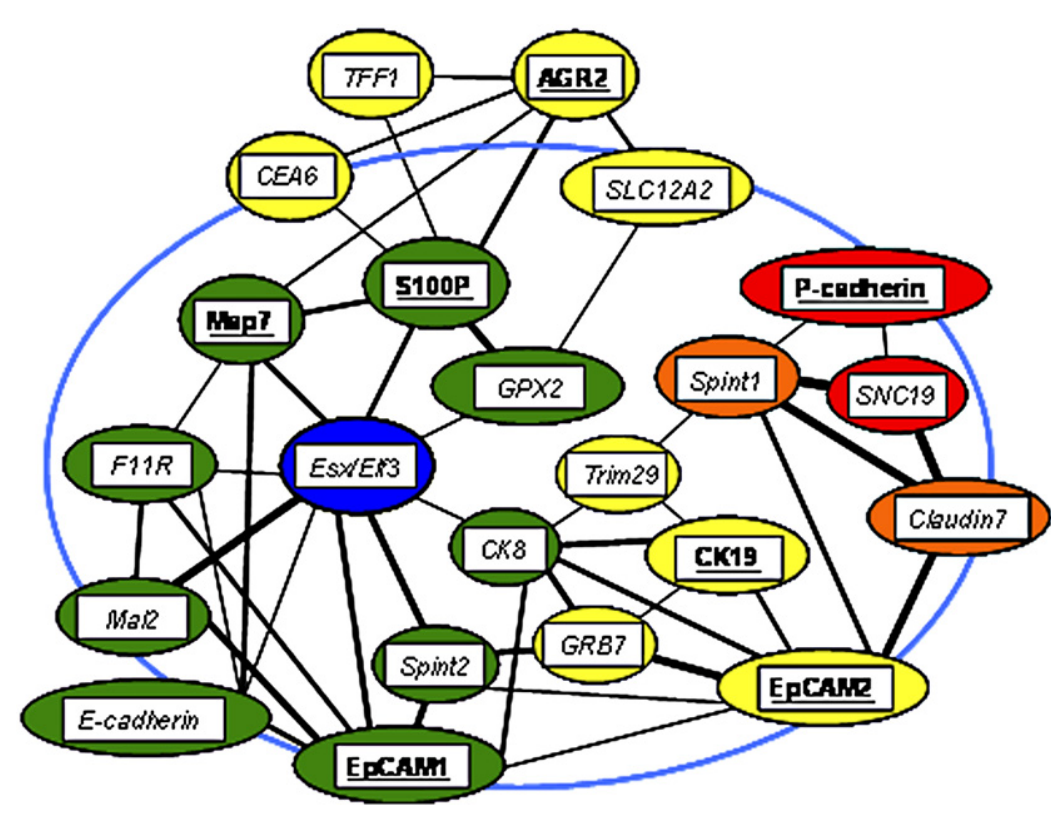

Figure 1. Correlation map of cancer-associated genes. Correlation map of the genes was constructed as described in the text. Genes are positioned in a hypothetical cell to reflect intracellular, membrane-bound, or extracellular localization. The thickness of a solid line connecting a given gene pair is $\sim$ proportional to the $R^{2}$ value of gene expression, which ranges from $0.91(P<.0001)$ for the Spint1/SNC19 pair, to $0.55(P<.0001)$ for the $T F F 1 / S 100 P$ pair. 
purchased from PE Biosystems. Standard reaction volume was 10 $\mu \mathrm{L}$ and contained $1 \mathrm{X}$ SYBR RT-PCR buffer, $3 \mathrm{mmol} / \mathrm{L} \mathrm{MgCl}_{2}$, $0.2 \mathrm{mmol} / \mathrm{L}$ each of deoxyadenosine triphosphate, deoxycytosine triphosphate, deoxyguanosine triphosphate, $0.4 \mathrm{mmol} / \mathrm{L}$ deoxyuridine triphosphate, 0.1 U UngErase enzyme, 0.25 U AmpliTaq Gold, $0.35 \mu \mathrm{L}$ cDNA template, and $50 \mathrm{nmol} / \mathrm{L}$ of oligonucleotide primer. Initial steps of RT-PCR were 2 minutes at $50^{\circ} \mathrm{C}$ for UngErase activation, followed by a 10 -minute hold at $95^{\circ} \mathrm{C}$. Cycles $(\mathrm{n}=$ 40) consisted of a 15 -second melt at $95^{\circ} \mathrm{C}$ followed by a 1 -minute annealing/extension at $60^{\circ} \mathrm{C}$. The final step was a $60^{\circ} \mathrm{C}$ intubation for 1 minute. All reactions were performed in triplicate. Threshold for cycle of threshold $(\mathrm{Ct})$ analysis of all samples was set at 0.5 relative fluorescence units.

Gene expression values were quantified as $\Delta \mathrm{Ct}$ values, which were obtained by subtracting the $\mathrm{Ct}$ value of an internal reference control gene ( $\beta 2$-microglobulin, B2M) from the gene of interest. $\mathrm{Ct}$ values are inversely proportional to gene expression levels and are based on $\log 2$ scale.

The results were internally validated by repeating the real-time RT-PCR process using a new section cut from tissue blocks of the primary tumor. Variability of tumor quantity on the sections was minimized by hematoxylin and eosin comparison performed by a pathologist. A cross-validation procedure was used to determine whether the results were sensitive to the samples included. A leave-one-out procedure was used whereby each sample was systemically removed and the data reanalyzed.

\section{Statistical Analysis}

To assess for prognostic accuracy, we analyzed receiver operating characteristic curves on the individual genes normalized to B2M (Med Calc Software, Mariakerke, Belgium). Prognostic gene combinations were tested by subtracting $\Delta \mathrm{Ct}$ values generated by RTPCR analysis. Subtraction of $\Delta \mathrm{Ct}$ values $(\Delta \Delta \mathrm{Ct})$ is equivalent to the $\log$ of the ratio of values. In the text, the $\Delta \mathrm{Ct}_{\text {gene } \mathrm{A}}-\Delta \mathrm{Ct}_{\text {gene }}$ B calculation is abbreviated as a gene expression ratio. The value of the 2-gene prognostic assay was further assessed by KaplanMeier survival analysis.

\section{Results}

A primary tumor's ability to metastasize requires many genetic events. In this study, we hypothesized that there are relatively few genes that may be critical to the metastatic phenotype, such that high expression of a gene that portends nonrecurrence coupled with the low expression of a gene critical to metastasis would be useful to predict clinical outcome in adenocarcinoma of the lung.

The correlation map illustrated in Figure 1 resulted from a unique bioinformatics analysis that led to a set of genes that had specific structured connections based on a query of 15 genes overexpressed in 4 lung cancer cell lines. Of the 22 identified genes, 7 were in the original query set and were labeled primary prognostic genes. These genes combined with 6 of the most frequently expressed remaining 16 secondary genes constituted the study's test gene set in patients with adenocarcinoma of the lung. This unique approach is somewhat similar to the description of expression profiles in different tumors in terms of behavior modules, sets of genes that are in concert to carry out a specific function. ${ }^{10}$ In fact, many of the genes in this study test set were contained in one of the modules (module 180) described by Segal and colleagues. $^{10}$

Area under the curve (AUC) values for the primary and secondary genes are shown in Table 2. Receiver operating characteristic curve analysis of the individual genes revealed that high expression of CK19 was associated with nonrecurrence ( $\geq 4$ years) (AUC $=0.859 ; 95 \%$ confidence interval $[\mathrm{CI}]=0.651-0.970)$; whereas high expression of EpCAM2 was associated with disease recurrence within 2 years (AUC $=0.606 ; 95 \% \mathrm{CI}=0.366-0.813$ ).

To determine whether the prognostic accuracy of CK19 could be improved by combining it with another gene whose overexpression might be necessary for the metastatic phenotype and therefore low expression be favorable, we subtracted the mean $\Delta \mathrm{Ct}$ values of individual genes as determined by real-time RT-PCR analysis from $\Delta \mathrm{Ct}_{\mathrm{CK} 19}$. For all potential CK19/gene X combinations, the ratio of CK19/EpCAM2 yielded the highest prognostic accuracy as determined by AUC measurements (Table 3). This observation provided evidence that EpCAM2 is a "bad" gene. The CK19/EpCAM2 expression ratio, which was derived from the mean of two experiments, also performed well when data were analyzed from individual experiments. In the first and second experiments, the prognostic accuracy of the CK19/EpCAM2 expression ratio as determined by AUC analysis was $0.91(95 \% \mathrm{CI}=0.69-0.99)$ and $0.84(95 \%$ $\mathrm{CI}=0.56-0.97)$, respectively (data not shown). Of further note is the observation that among the 12 patients with stage I adenocarcinoma, the prognostic accuracy of the CK19/ EpCAM2 expression ratio was 92\% (11/12).

TABLE 2. Recurrence analysis of pilot study using single markers paired with the internal $B 2 M$ reference control gene

\begin{tabular}{lcc}
\hline & \multicolumn{2}{c}{ Recurrence analysis } \\
\cline { 2 - 3 } \multicolumn{1}{c}{ Gene } & AUC & 95\% CI \\
\hline CK19 & 0.859 & $0.631-0.970$ \\
EpCAM2 & 0.606 & $0.366-0.813$ \\
AGR2 & 0.596 & $0.357-0.805$ \\
Esx & 0.566 & $0.329-0.782$ \\
GPX2 & 0.556 & $0.320-0.773$ \\
CEA6 & 0.545 & $0.312-0.765$ \\
E-cadherin & 0.545 & $0.312-0.765$ \\
EpCAM1 & 0.535 & $0.303-0.757$ \\
SPINT2 & 0.530 & $0.298-0.753$ \\
S100P & 0.525 & $0.294-0.749$ \\
MAL2 & 0.515 & $0.285-0.740$ \\
P-cadherin & 0.510 & $0.281-0.736$ \\
Map7 & 0.500 & $0.272-0.728$ \\
UPAR & 0.470 & $0.247-0.702$ \\
\hline AUC Ara &
\end{tabular}

$A U C$, Area under the curve; $C l$, confidence interval. 
TABLE 3. Recurrence and survival analysis of pilot study based on CK19/gene $X$ ratios

\begin{tabular}{|c|c|c|c|c|}
\hline \multirow[b]{2}{*}{ GeneX } & \multicolumn{2}{|c|}{ Recurrence analysis* } & \multicolumn{2}{|c|}{$\begin{array}{c}\text { Kaplan-Meier } \\
\text { survival analysis } \dagger\end{array}$} \\
\hline & AUC & $95 \% \mathrm{CI}$ & $P$ value & HR \\
\hline EpCAM2 & 0.879 & $0.656-0.978$ & .0001 & 10.7 \\
\hline P-cadherin & 0.874 & $0.650-0.976$ & .0003 & 8.13 \\
\hline MAL2 & 0.869 & $0.643-0.974$ & .0004 & 9.24 \\
\hline Esx & 0.742 & $0.501-0.908$ & .0008 & 6.62 \\
\hline Map7 & 0.889 & $0.668-0.981$ & .0013 & 6.24 \\
\hline UPAR & 0.843 & $0.613-0.963$ & .0013 & 6.24 \\
\hline E-cadherin & 0.818 & $0.584-0.951$ & .0013 & 6.25 \\
\hline AGR2 & 0.859 & $0.631-0.970$ & .0098 & 4.69 \\
\hline GPX2 & 0.722 & $0.480-0.895$ & .0184 & 5.12 \\
\hline SPINT2 & 0.848 & $0.619-0.965$ & .0207 & 7.78 \\
\hline EpCAM1 & 0.798 & $0.561-0.940$ & .0207 & 7.78 \\
\hline S100P & 0.732 & $0.490-0.901$ & .0275 & 4.08 \\
\hline CEA6 & 0.732 & $0.490-0.901$ & .0729 & 3.10 \\
\hline
\end{tabular}

$A U C$, Area under the curve; $C l$, confidence interval; $H R$, hazard ratio. *AUC based on continuous representation of gene expression values. †Hazard ratios based on binary analysis of best thresholds of outcome discrimination.

The cross-validation procedure found no qualitative differences in inferences. For CK19 alone, the range of AUCs found in the cross-validation analyses was (0.87 to 0.92 ) whereas the AUC when all samples were included was 0.86. Analogous results were found when CK19 was combined with EpCAM2.

To further assess the value of CK19 unpaired and paired with EpCAM2, we performed a Kaplan-Meier survival analysis using data generated from single marker and CK19/gene $\mathrm{X}$ analyses. For the single CK19 marker, a $\Delta \mathrm{Ct}$ cutoff of 11.4 was used, which separated the 20 patients into high $(\Delta \mathrm{Ct}<$ $11.4 ; \mathrm{n}=13)$ and low $(\Delta \mathrm{Ct}>11.4 ; \mathrm{n}=7)$ expressing tumors. A log-ranked test indicated that the two curves generated as a function of marker positivity were different at a $P$ value of .0021 with a hazard ratio of 6.2 (Figure 2,
A). For the CK19/EpCAM2 ratio, a $\Delta \Delta \mathrm{Ct}$ cutoff of 7.2 was used, which separated the 20 patients into high $(\Delta \Delta \mathrm{Ct} \leq$ $7.2 ; \mathrm{n}=13)$ and low $(\Delta \Delta \mathrm{Ct}>7.2 ; \mathrm{n}=7)$ groups that correlated with survival. A log-ranked test indicated that the two curves generated as a function of marker positivity were different at a $P$ value of .0001 with an associated hazard ratio of 10.7 (Figure 2, B). Kaplan-Meier survival analysis of other CK19/gene X pairs is shown in Table 3. The gene pair that yielded the second most highly significant curves was CK19/P-cadherin, with an associated hazard ratio of 8.1.

To determine assay reliability, we applied the 2 -gene test to a set of patients $(n=22)$ who were treated at the Mayo Clinic, Jacksonville, Florida. Twelve patients survived longer than 2 years, whereas 10 patients had recurrence within 2 years. All patients in this data set died by 65 months. We observed that the hazard ratio of CK19/EpCAM2 expression pair decreased to 4.5 but remained significant $(P=.007)$. The CK19/P-cadherin expression ratio also clearly identified patients with longer survival (hazard ratio $=3.24 ; P=.0029$ ).

\section{Discussion}

Unfortunately, a large number of patients with resected earlystage lung cancer will have a recurrence within 2 to 3 years. The ability to predict those patients at high risk for recurrence could help direct the possible addition of therapy to improve survival and, vice versa, avoid the toxicity for those at low risk. Many molecular markers that predict patient survival independent of TNM status have been reported. ${ }^{11}$ Tools used to predict recurrence have included immunohistochemical analysis, ${ }^{12}$ cDNA microarray profiling, ${ }^{13-17}$ real-time RT-PCR, ${ }^{6,18-20}$ and most recently, proteomics. ${ }^{21}$ Many of the methods have been costly, not readily available to the average surgeon, required frozen tissue specimens, and have therefore been difficult to translate from the research laboratory to the clinical arena.

In the present study, we measured the expression of 14 different test genes and one internal reference control gene
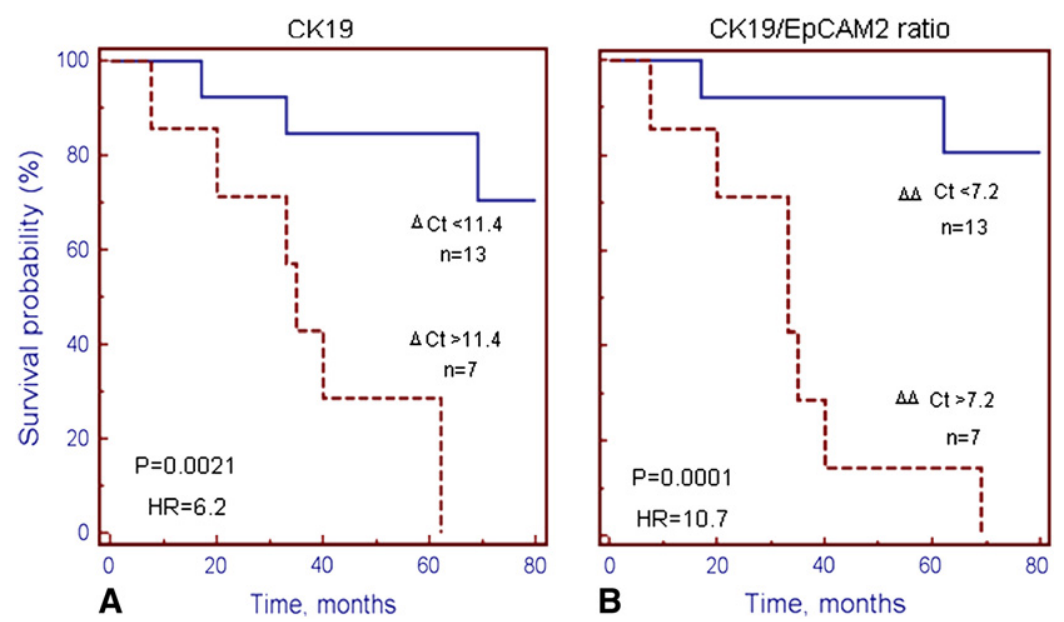

The Journal of Thoracic and Cardiovascular Surgery • Volume 135, Number 3
Figure 2. Kaplan-Meier survival analysis. Data generated from single-marker (A) and CK19/ EpCAM2 (B) analyses. HR, Hazard ratio. 
in primary tumors resected from patients with early-stage NSCLC. Using the B2M gene as an internal reference, we observed that high expression of CK19 was correlated with good clinical outcome (no disease recurrence), whereas high expression of EpCAM2 was correlated with poor clinical outcome (disease recurrence within 2 years). Of all possible 2-gene combinations $(n=105)$, we further observed that the ratio of CK19/EpCAM2 had the highest accuracy for predicting disease recurrence. The concept of using a 2-gene ratio was previously applied to NSCLC by Gordon and coworkers, ${ }^{19,22}$ who identified S100P as 1 of 7 prognostic markers. It should be noted that in the Mayo data set, the marker combination of CK19/S100P yielded results similar to CK19/P-cadherin (data not shown). However, the current study is the first to analyze the expression of genes in paraffin samples. In colon cancer, high expression of EpCAM2 (also known as TROP2) has been shown to be associated with a higher frequency of liver metastasis $(P=.005)$ and more cancer-related deaths $(P=.046),{ }^{23}$ a finding that further supports the concept that for early-stage NSCLC, EpCAM2 is a "bad gene."

The gene pair with the second highest prognostic accuracy for disease recurrence was CK19/P-cadherin. Previous studies have shown that expression levels of P-cadherin in primary tumors correlate with tumor grade in ovarian cancer ${ }^{24}$ and metastases to the lung in thyroid cancer. ${ }^{25}$ Further, overexpression of P-cadherin in vitro results in increased cell motility in pancreatic cancer, ${ }^{26}$ a necessary requirement for establishment of distant metastases. Taken together, these results provide evidence that $\mathrm{P}$-cadherin may also serve as a candidate "bad gene" in NSCLC. Regarding CK19, antibodies to the protein encoded by this gene (and/or a combination of other cytokeratin genes) have been used for the detection of circulating tumor cells in breast, lung, colon, and other cancers. ${ }^{27,28}$ In the current study, we suspect that CK19 expression levels serve as a reliable indicator of the epithelial content of the primary tumor.

Although there was a recent report of the use of real-time RT-PCR for prognosis of patients with early-stage NSCLC, the current study differs significantly from the approach taken by Chen and colleagues. ${ }^{20}$ In this report, patient prognosis was based on a simple calculation of a 2-gene ratio, an approach that contains only one "decision node." In the study of Chen and associates, a 5-gene marker panel was used that required a relatively high number of decision nodes $(n=19)$. An algorithm that uses such a high number of decision nodes for a low number of genes is less likely to be clinically applicable because of its cumbersome nature. In contrast, the microarray study of Potti and coworkers ${ }^{6}$ required only 5 decision nodes, even though 289 genes were involved.

There are several advantages to the technique used in this preliminary study. It is a simple 2-gene model and uses a technology that is relatively inexpensive and is quickly per- formed once RNA is extracted. Paraffin-embedded tumor tissue can be screened and an appropriate slide(s) could be sent to a reference laboratory. The technique is amenable to small tissue samples, which may be important if preoperative biopsy directs neoadjuvant therapy.

Several limitations of this pilot analysis need to be acknowledged. First, given the small numbers used for the preliminary study, external verification must be performed on a larger data set before definitive statements are made concerning its application as a prognostic tool. Second, given the number of putative genes that could display either a direct or inverse relationship between expression and prognosis, it is possible that another gene ratio or a combination of two ratio sets will be more informative as patients are added. Correlative experiments looking at protein levels in tumor issues should be a future goal.

In summary, a simple 2-gene molecular model has been developed to predict recurrence in patients having resection of early-stage adenocarcinoma of the lung. The model will require further validation and refinement. It is hoped that in the future a relatively easy, cost-effective, clinically relevant molecular model will be used to individualize therapy in early-stage NSCLC.

\section{References}

1. Mountain CF. Revisions in the international system for staging lung cancer. Chest. 1997;11:1710-7.

2. The International Adjuvant Lung Cancer Trial Collaborative Group. Cisplatin-based adjuvant chemotherapy in patients with completely resected non-small-cell lung cancer. $N$ Engl J Med. 2004;350:351-60.

3. Winton T, Livingston R, Johnson D, Rigas J, Johnston M, Butts C, et al. Vinorelbine plus cisplatin vs. observation in resected non-small cell lung cancer. N Engl J Med. 2005;352:2589-97.

4. Strauss G, Herndon J, Maddaus M, Johnstone DW, Johnson EA, Watson DM, et al. Adjuvant chemotherapy in stage IB non-small cell lung cancer (NSCLC): update of cancer and leukemia group B (CALGB) protocol 9633. Proc Am Soc Clin Oncol. 2006;24:365s.

5. Pignon J, Tribodet H, Scagliotti G, Douillard JY, Shepherd FA, Stephens RJ, et al. Lung adjuvant cisplatin evaluation (LACE): a pooled analysis of five randomized clinical trials including 4,584 patients. Proc Am Soc Clin Oncol. 2006;24:366s.

6. Potti A, Mukherjee S, Petersen R, Dressman HK, Bild A, Koontz J, et al. A genomic strategy to refine prognosis in early-stage non-small cell lung cancer. $N$ Engl J Med. 2006;355:570-80.

7. Bertucci F, Finetti P, Cervera N, Maraninchi D, Viens P, Birnbaum D. Gene expression profiling and clinical outcome in breast cancer. OMICS. 2006;10:429-43.

8. Mikhitarian K, Gillanders WE, Almeida JS, Herbert-Martin R, Varela JC, Metcalf JS, et al. An innovative microarray strategy identifies informative molecular markers for the detection of micrometastatic breast cancer. Clin Cancer Res. 2005;11. C-704.

9. Sprecht K, Richter T, Mueller U, Walch A, Werner M, Hofler H. Quantitative gene expression analysis in microdissected archival formalin-fixed and paraffin-embedded tumor tissue. Am J Pathol. 2001;158:419-29.

10. Segal E, Friedman N, Koller D, Regev A. A module map showing conditional activity of expression modules in cancer. Nat Genet. 2004; 36:1090-8.

11. Brundage MD, Davies D, Mackillop WJ. Prognostic factors in nonsmall cell lung cancer: a decade of progress. Chest. 2002;122:1037-57.

12. D'Amico TA, Massey M, Herndon JE, Moore M-B, Harpole DH. A biologic risk model for stage I lung cancer: immunohistochemical analysis 
of 408 patients with the use of ten molecular markers. J Thorac Cardiovasc Surg. 1999;117:736-43.

13. Garber ME, Troyanskaya OG, Schluens K, Petersen S, Taesher Z, Pacyna-Gengelbach $\mathrm{M}$, et al. Diversity of gene expression in adenocarcinoma of lung. Proc Natl Acad Sci U S A. 2001;98:13784-9.

14. Chen C, Gharib TG, Huang C-C, Kuick R, Thomas DG, Shedden KA, et al. Protein profiles associated with survival in lung adenocarcinoma. Proc Natl Acad Sci U S A. 2003;100:13537-42.

15. Bhattacharjee A, Richards WG, Staunton J, Li C, Monti S, Vasa P, et al. Classification of human lung carcinomas by mRNA expression profiling reveal distinct adenocarcinoma subclasses. Proc Natl Acad Sci U S A. 2001;98:13790-5.

16. Wigle DA, Jurisica I, Radulovich N, Pintilie M, Rossant J, Liu N, et al Molecular profiling of non-small cell lung cancer and correlation with disease-free survival. Cancer Res. 2002;62:3005-8.

17. Yanaihara N, Caplen N, Bowman E, Seike M, Kumamoto K, Yi M, et al. Unique microRNA molecular profiles in lung cancer diagnosis and prognosis. Cancer Cell. 2006;9:189-98.

18. Endoh H, Tomida S, Yatabe Y, Konishi H, Osada H, Tajima K, et al. Prognostic model of pulmonary adenocarcinoma by expression profiling of eight genes as determined by quantitative real-time reverse transcriptase polymerase chain reaction. J Clin Oncol. 2004;22:811-9.

19. Gordon GJ, Jensen RV, Hsiao L-L, Gullans SR, Blumenstock JE, Ramaswamy S, et al. Translation of microarray data into clinically relevant cancer diagnostic tests using gene expression ratios in lung cancer and mesothelioma. Cancer Res. 2002;62:4963-7.

20. Chen HY, Yu SL, Chen CH, Chang GC, Chen CY, Yuan A, et al. A five gene signature and clinical outcome in non-small cell lung cancer. N Engl J Med. 2007;356:11-20.

21. Kikuchi T, Carbone DP. Proteomic analysis in lung cancer: challenges and opportunities. Respirology. 2007;12:22-8.

22. Gordon GJ, Richards WG, Sugarbaker DJ, Jaklitsch MT, Bueno R. A prognostic test for adenocarcinoma of the lung from gene expression profiling data. Cancer Epidemiol Biomarkers Prev. 2003;12:905-10.

23. Ohmachi T, Taneka F, Mimori K, Inoue H, Yanaga K, Mori M. Clinical significance of TROP2 in colorectal cancer. Clin Cancer Res. 2006;12: 3057-63.

24. Patel IS, Madan P, Getsios S, Bertrand MM, MacCalman CO. Cadherin switching in ovarian cancer progression. Int J Cancer. 2003;106:172-7.

25. Zou M, Famulski KS, Parhour RS, Baitei E, Al-Mohann FA, Farid NR, et al. Microarray analysis of metastasis-associated gene expression profiling in a murine model of thyroid carcinoma pulmonary metastasis: identification of S100A4 (Mts 1) gene over expression as a poor prognostic marker for thyroid carcinoma. J Clin Endocrinol Metab. 2004;89:61646-54.

26. Taniuchi K, Nakagawa H, Hosokawa M, Nakamura T, Eguchi H, Ohigashi $\mathrm{H}$, et al. Over expressed $\mathrm{P}$-cadherin/CDH3 promotes motility of pancreatic cancer cells by interacting with p120ctn and activating rho-family GTPases. Cancer Res. 2005;65:3092-9.

27. Allard WJ, Matera J, Miller MC, Repollet M, Connelly MC, Rao C, et al. Tumor cells circulate in the peripheral blood of all major carcinomas but not in healthy subjects of patients with nonmalignant diseases. Clin Cancer Res. 2004;10:6897-904.

28. Cristofanilli M, Budd GT, Ellis MJ, Stopeck A, Matera J, Miller MC, et al. Circulating tumor cells, disease progression, and survival in metastatic breast cancer. $N$ Engl J Med. 2004;351:781-91.

\section{Discussion}

Dr Virginia R. Litle (New York, $N Y$ ). Dr Reed, you have addressed a very important clinical question: Which patients with early-stage lung cancer may benefit from adjuvant therapy because they are at higher risk of recurrent disease. You have tried to keep it simple and straightforward and emphasize that. However, I have a few questions about the research design.

In this study you compared gene expression in the tumors versus the normal tissue. Often this may be better suited to identifying diagnostic markers rather than prognostic markers. You compared gene expression in the tumor cell lines with that in normal lymph nodes. This approach would typically identify potential markers for detection of lymph node metastases. It is not clear to me why this would identify markers that may be predictive of prognosis for poor outcome. Maybe you could clarify the logic of this approach.

Following up on that, in your table for recurrence analysis of single markers, the AUC was greater than 0.6 for CK19 only. For all the other markers, it was less than 0.6. How do we know identification of these markers might only be occurring by chance?

Dr Reed. The idea here was to try to simplify the process, as you noted, and the study actually started by looking at the ability to predict recurrence on lymph nodes gathered by very minimally invasive ways. After a series of studies, it became apparent that if the hypothesis was to predict the metastatic phenotype, we should be looking at the primary tumor itself rather than looking at lymph nodes. We went back to look at the primary tumors and found that correlated much better with the metastatic phenotype than looking at normal tissue. Therefore, through a series of algorithms and using the database, we then looked at those genes that were connected with each other in metastatic disease. It turns out that there have been other studies to verify that it looks like EpCAM2, particularly, is a molecule that may be a causal link to metastases. I think your question concerns what is diagnostic and what is prognostic, and our study is really trying to identify those patients whom we could test up front through looking at their primary tumors to determine the likelihood of recurrence. Thus our focus has been really prognostic, not diagnostic. That does not mean the efforts that we have used and the type of things we are doing could not be potentially used in a diagnostic manner. That is actually the way we started out, trying to look at early diagnosis and prognosis of metastasis with the lymph nodes gathered through minimally invasive approaches, and, frankly, it did not pan out.

Dr Litle. Are you confident that you will be able to validate this on a separate sample set?

Dr Reed. That is a good question. We already have validated it on a separate set of patients who were supplied to us by our associates at Mayo-Jacksonville. In one of the conclusions, I was careful to say that the CK19/EpCAM2 ratio may be a prognostic indicator. The other ratio that may be very useful is the CK19/P/cadherin ratio. I did not have time to look at my cancer-associated map, which is pretty complex. However, if we look at the connection of these genes, it appears that there is a transcription gene at the center of this map that may be a molecular target for a drug, and that is our ultimate goal.

Dr Litle. What do you think is the biological or functional rationale behind CK19 being involved as a poor prognostic factor?

Dr Reed. Well, CK19 is not a poor prognosticator. It is a good prognosticator.

Dr Litle. Excuse me, a good prognostic factor.

Dr Reed. It is a cytoskeletal protein. I do not know the answer to that. It obviously remains intact, and so when we put it up with the other ratios of the other genes, it turned out to be very prognostic. I cannot answer that question with any great expertise.

Dr Raphael Bueno (Boston, Mass). I am delighted that you are presenting these results. We published this concept in mesothelioma and lung cancer in 2002 in a cover article of Cancer Research, validated it in mesothelioma in the Journal of the National Cancer 
Institute, did the same thing in adenocarcinoma of the lung in terms of early prognosis in 2004, and published on it extensively again. I am pleased that you have provided us with external validation of our work.

I can answer some of the questions that Dr Litle asked, as we have been studying this method using National Institutes of Health grants. This method provides for a signal-to-noise ratio, and that is why it is so powerful. In addition, the genes used here may have absolutely no biological meaning. These genes' overexpression may result from a downstream event that may be mechanistically meaningful or not. One of the prognostic genes in poor-outcome adenocarcinomas that we have reported is the glucose transporter that lets the F-deoxyglucose get into the cells for "lighting up" positron emission tomographic scans, for example, which is consistent with other data about that glucose transporter gene expression. We also found that it is best at the initial comparative set to identify the genes and then refine them in the training set. As we have been doing this in lung cancer, mesothelioma, prostate cancer, and other cancers, we found that actually a combination of two or three ratios gives us a much better specificity and sensitivity.

I hope that you continue to work on it and be successful. I would like to declare that I do have a slight conflict of interest as my institution has a patent pending on this technology.

Dr David R. Jones (Charlottesville, $\mathrm{Va}$ ). You looked at overexpressed genes. As you know, there are many genes that in fact are suppressors of metastasis and there is a loss of these genes. Have you thought about going back and looking at maybe the top 15 genes that are underexpressed to determine whether there is a difference?

Second, what is the biology of EpCAM2, or how does its signaling really work, for those of us who are not as familiar with that?

Dr Reed. The reason we looked at overexpressed genes was because of the RT-PCR that we used. We believed that if we looked at overexpressed genes, the sensitivity would be much higher. That is a very good point, but that was the reason that we looked at overexpressed genes.

EpCAM2 (also know as TROP2) is a molecule that is believed to be involved in hemotypic intracellular adhesion of epithelial cells, signal transduction. There has been a very recent presentation at the American Association of Cancer Research meeting to show that EpCAM2 particularly is overexpressed in a large number of metastases, from colon, stomach, breast, and ovary tumors and may very well be a novel widespread stimulator of cancer growth. There is a lot of information actually that EpCAM2 is an important gene for metastasis.

Dr Jack A. Roth (Houston, Tex). It is a very nice study. Have you looked at the protein levels with immunohistochemistry? Do they correlate with your RNA levels?

Dr Reed. The team at Mayo Clinic-Jacksonville is doing that now. We have given them our correlative slides to do that study. 